\title{
The Transit Light Curve (TLC) Project. VI. Three Transits of the Exoplanet TrES\#2
}

\section{Citation}

Holman, Matthew J., Joshua N. Winn, David W. Latham, Francis T. O'Donovan, David Charbonneau, Guillermo Torres, Alessandro Sozzetti, Jose Fernandez, and Mark E. Everett. 2007. "The Transit Light Curve (TLC) Project. VI. Three Transits of the Exoplanet TrES\#2." The Astrophysical Journal 664 (2): 1185-89. https://doi.org/10.1086/519077.

\section{Permanent link}

http://nrs.harvard.edu/urn-3:HUL.InstRepos:41397454

\section{Terms of Use}

This article was downloaded from Harvard University's DASH repository, and is made available under the terms and conditions applicable to Other Posted Material, as set forth at http:// nrs.harvard.edu/urn-3:HUL.InstRepos:dash.current.terms-of-use\#LAA

\section{Share Your Story}

The Harvard community has made this article openly available. Please share how this access benefits you. Submit a story.

Accessibility 


\title{
THE TRANSIT LIGHT CURVE (TLC) PROJECT. VI. THREE TRANSITS OF THE EXOPLANET TrES-2 \\ Matthew J. Holman, ${ }^{1}$ Joshua N. Winn, ${ }^{2}$ David W. Latham, ${ }^{1}$ Francis T. O’Donovan, ${ }^{3}$ David Charbonneau, ${ }^{1,4}$ Guillermo Torres, ${ }^{1}$ Alessandro Sozzetti, ${ }^{1,5}$ Jose Fernandez, $^{1}$ and Mark E. Everett ${ }^{6}$ Received 2007 March 20; accepted 2007 April 20
}

\begin{abstract}
Of the nearby transiting exoplanets that are amenable to detailed study, TrES-2 is both the most massive and the one with the largest impact parameter. We present $z$-band photometry of three transits of TrES- 2 . We improve on the estimates of the planetary, stellar, and orbital parameters, in conjunction with the spectroscopic analysis of the host star by Sozzetti and coworkers. We find the planetary radius to be $R_{p}=1.222 \pm 0.038 R_{\text {Jup }}$ and the stellar radius to be $R_{\star}=1.003 \pm 0.027 R_{\odot}$. The quoted uncertainties include the systematic error due to the uncertainty in the stellar mass $\left(M_{\star}=0.980 \pm 0.062 M_{\odot}\right)$. The timings of the transits have an accuracy of $25 \mathrm{~s}$ and are consistent with a uniform period, thus providing a baseline for future observations with the NASA Kepler satellite, whose field of view will include TrES-2.
\end{abstract}

Subject headings: planetary systems — stars: individual (GSC 03549-02811) — techniques: photometric Online material: machine-readable table

\section{INTRODUCTION}

Careful follow-up observations of nearby transiting planet systems have revolutionized our understanding of a whole new kind of planet: hot Jupiters. They have been used to reveal absorption by atmospheric atomic sodium (Charbonneau et al. 2002) and the presence of an extended hydrogen exosphere (Vidal-Madjar et al. 2003) in HD 209458b, as well as to detect the thermal infrared emission from TrES-1, HD 209458b, and HD 189733b (Charbonneau et al. 2005; Deming et al. 2005, 2006). They have been used to investigate the spin-orbit alignment of HD 209458b (Queloz et al. 2000; Winn et al. 2005) and HD 189733b (Winn et al. 2006). Most recently, spectra of the infrared planetary emission of HD $189733 \mathrm{~b}$ (Grillmair et al. 2007) and HD 209458b (Richardson et al. 2007), obtained with the Spitzer Space Telescope, have been used to constrain models of the atmospheric content of those planets.

Through these observations we are steadily improving our understanding of the interior and atmospheric structure of hot Jupiters. Future measurements, such as reflected-light observations or the detection of other atmospheric constituents through transmission spectroscopy, will continue to advance our knowledge of these planets. One goal of the Transit Light Curve (TLC) project is to support these efforts by refining the estimates of the planetary, stellar, and orbital parameters through high-accuracy, high-cadence photometry of exoplanetary transits. We also seek to measure or bound any variations in the transit times and lightcurve shapes that would be caused by the influence of additional bodies in the system (Miralda-Escudé 2002; Agol et al. 2005; Holman \& Murray 2005). Along the way, we are exploring different techniques for photometry and parameter determination. Previous papers in this series have reported results for the exoplanets XO-1b (Holman et al. 2006), OGLE-TR-111b (Winn

\footnotetext{
1 Harvard-Smithsonian Center for Astrophysics, Cambridge, MA 02138; mholman@cfa.harvard.edu.

2 Department of Physics and Kavli Institute for Astrophysics and Space Research, Massachusetts Institute of Technology, Cambridge, MA 02139.

3 California Institute of Technology, Pasadena, CA 91125.

4 Alfred P. Sloan Research Fellow.

5 INAF-Osservatorio Astronomico di Torino, 10025 Pino Torinese, Italy.

6 Planetary Science Institute, Tucson, AZ 85719.
}

et al. 2007a), TrES-1 (Winn et al. 2007b), OGLE-TR-10b (Holman et al. 2007), and HD 189733b (Winn et al. 2007c).

The present paper is concerned with TrES-2, the second transiting hot Jupiter discovered by the Transatlantic Exoplanet Survey (O'Donovan et al. 2006). The planet orbits a nearby G0 V star (GSC 03549-02811) and transits every $\sim 2.5$ days. Although each of the 14 known transiting exoplanets has its own story to tell (see Charbonneau et al. 2007 for a review), the TrES-2 system has at least three distinguishing characteristics.

First, TrES-2 is the first transiting extrasolar planet discovered in the field of view of the NASA Kepler mission (Borucki et al. 2003; Basri et al. 2005). Kepler will observe nearly 600 transits of TrES-2 during the nominal 4 yr lifetime of the mission. This opportunity prompts us to improve the determinations of the orbital parameters of TrES-2 for comparison to the future estimates from Kepler.

Second, TrES-2 has the highest impact parameter of any known nearby transiting extrasolar planet. This makes the duration of the transit (as well as the duration of ingress and egress) more sensitive to changes in impact parameter. This, in turn, makes TrES-2 an excellent target for the detection of long-term changes in transit characteristics induced by orbital precession (Miralda-Escudé 2002).

Third, the mass of TrES-2 is the largest of the known nearby transiting extrasolar planets. Furthermore, the radius of TrES-2 appears somewhat larger than predicted by simple structural models of irradiated hot Jupiters, as also appears to be the case for HAT-P-1b, WASP-1b, and HD 209458b (although see Burrows et al. [2007] for a contrary view).

In what follows we present TLC results for TrES-2. In $\S 2$ we describe the observations and the data reduction procedures. In $\S 3$ we describe the model and techniques we used to estimate the physical and orbital parameters of the TrES-2 system, and in $\S 4$ we summarize our results.

\section{THE OBSERVATIONS AND DATA REDUCTION}

We observed four transits of TrES-2. According to the ephemeris provided by O'Donovan et al. (2006),

$$
T_{c}(E)=2,453,957.6358(\mathrm{HJD})+E(2.47063 \text { days }),
$$



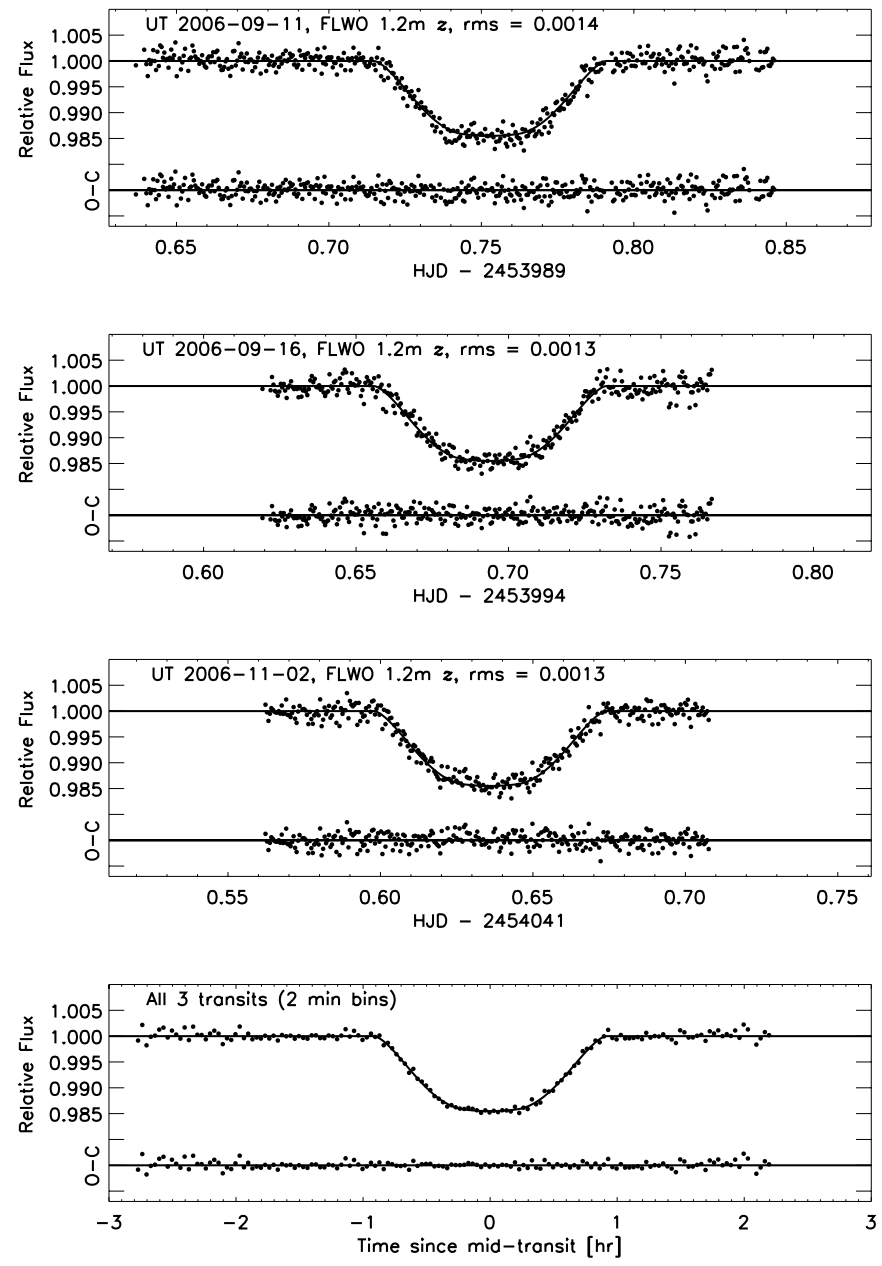

FIG. 1.-Photometry of TrES-2 in the $z$ band, using the FLWO $1.2 \mathrm{~m}$ telescope and KeplerCam. These data were used to estimate the planetary, stellar, and orbital parameters (see $\S 3$ ). The bottom panel is a composite light curve created from the three data sets, after time shifting and averaging into 2 minute bins. The residuals (observed - calculated) are plotted beneath the data.

these transits correspond to epochs $13,15,32$, and 34 on UT 2006 September 11, September 16, October 28, and November 2 , respectively. Observations of a fifth transit, epoch 17 , were scheduled but were not executed due to poor weather.

We observed these transits with KeplerCam on the $1.2 \mathrm{~m}$ (48 inch) telescope of the Fred L. Whipple Observatory (FLWO) on Mt. Hopkins, Arizona. This camera (PI: D. Latham) was built for a photometric survey of the target field of the Kepler satellite mission (Borucki et al. 2003). It has a single $4 \mathrm{~K} \times 4 \mathrm{~K}$ Fairchild $486 \mathrm{CCD}$ with a $23.1^{\prime} \times 23.1^{\prime}$ field of view. We used $2 \times 2$ binning, for which the readout and reset time is $11.5 \mathrm{~s}$ and the typical read noise is $7 e^{-}$per binned pixel. The response of each amplifier deviates from linearity by less that $0.5 \%$ over the range of counts from the faintest to brightest comparison star. We observed through the SDSS $z$ filter, the reddest available band, in order to minimize the effect of color-dependent atmospheric extinction on the relative photometry, and to minimize the effect of limb-darkening on the transit light curve.

The FWHM of a stellar image was typically $\sim 3$ binned pixels $\left(2^{\prime \prime}\right)$ on September 11, September 16, and November 2; the FWHM ranged from $\sim 3$ to $\sim 8$ pixels on October 28 . We used automatic guiding to maintain the locations of TrES-2 and its comparison stars to within a few pixels over the course of each night. We repeatedly took $30 \mathrm{~s}$ exposures for $3.5-5 \mathrm{hr}$ bracketing the predicted transit midpoint. The conditions on UT 2006
TABLE 1

Relative Рhotometry of TrES-2

\begin{tabular}{|c|c|c|}
\hline HJD & Relative Flux & Uncertainty \\
\hline $2,453,989.63669 \ldots$. & 0.9992 & 0.0013 \\
\hline $2,453,989.63943 \ldots \ldots \ldots \ldots \ldots \ldots \ldots \ldots \ldots \ldots \ldots$ & 1.0022 & 0.0013 \\
\hline $2,453,989.64013 \ldots \ldots \ldots \ldots \ldots \ldots \ldots \ldots$ & 0.9994 & 0.0013 \\
\hline $2,453,989.64058 \ldots \ldots \ldots \ldots \ldots \ldots \ldots \ldots$ & 0.9971 & 0.0013 \\
\hline $2,453,989.64105 \ldots \ldots \ldots \ldots \ldots \ldots \ldots \ldots \ldots \ldots \ldots \ldots \ldots \ldots \ldots$ & 1.0002 & 0.0013 \\
\hline $2,453,989.64150 \ldots \ldots \ldots \ldots \ldots \ldots \ldots \ldots \ldots \ldots \ldots \ldots \ldots \ldots \ldots \ldots$ & 0.9994 & 0.0013 \\
\hline
\end{tabular}

Notes.-Table 1 is published in its entirety in the electronic edition of the Astrophysical Journal. A portion is shown here for guidance regarding its form and content. The time stamps represent the Heliocentric Julian Date at the time of midexposure. The uncertainty estimates are based on the procedures described in $\S 2$.

September 11 were clear during the time of the observations, and the images were taken through air masses ranging from 1.05 to 1.90. The conditions on UT 2006 September 16 were also clear, and the air mass ranged from 1.05 to 1.40 . There were clouds passing overhead during the observations on UT 2006 October 28 , and the air mass ranged from 1.05 to 2.50 . The observing conditions were significantly worse during and after egress; the result was essentially to obtain observations of only a partial transit. Consequently, the data from October 28 were not included in the analysis below. There were very thin clouds during the observations on UT 2006 November 2, and the air mass ranged from 1.15 to 1.95 .

The images were calibrated using standard $\mathrm{IRAF}^{7}$ procedures for the overscan correction, trimming, bias subtraction, and flatfield division. We did not attempt to correct the fringing that was apparent with the $z$ filter. The fringing had a small amplitude and little effect on the final photometry, given the accuracy of the automatic guiding. We then performed aperture photometry of TrES-2 and 20 nearby comparison stars, using an aperture radius of 8.0 pixels $\left(4.3^{\prime \prime}\right)$ for each night. We subtracted the underlying contribution from the sky, after estimating its brightness within an annulus ranging from 30 to 35 pixels in radius, centered on each star. We divided the flux of TrES-2 by the total flux of the comparison stars.

To estimate the uncertainties in our photometry, we computed the quadrature sum of the errors due to Poisson noise of the stars (both TrES-2 and the comparison stars), Poisson noise of the sky background, readout noise, and scintillation noise (as estimated according to the empirical formulas of Young 1967 and Dravins et al. 1998). The dominant term is the Poisson noise from TrES-2. The final time series is plotted in Figure 1 and is available in machine-readable form in the electronic version of Table 1. (In that table, the quoted errors have been rescaled such that $\chi^{2} / N_{\text {dof }}=1$ for the best-fitting model, as explained in the next section.)

\section{DETERMINATION OF SYSTEM PARAMETERS}

Our methodology for determining the system parameters has been described in previous TLC papers (Holman et al. 2006; Winn et al. 2007a, 2007b, 2007c; Holman et al. 2007) and is summarized here. We assume a circular orbit of a planet (mass $M_{p}$, radius $R_{p}$ ) and a $\operatorname{star}\left(M_{\star}, R_{\star}\right)$, with period $P$ and inclination $i$ relative to the sky plane. We allow each transit to have an independent value of $T_{c}$ (the transit midpoint), rather than forcing

\footnotetext{
${ }^{7}$ IRAF is distributed by the National Optical Astronomy Observatory, which is operated by the Association of Universities for Research in Astronomy (AURA), Inc., under cooperative agreement with the National Science Foundation.
} 
them to be separated by exact multiples of the orbital period. Thus, the only effect of $P$ on the model is to determine the semimajor axis $a$ for a given value of $M_{\star}+M_{p}$. We fixed $P=$ 2.47063 days (O'Donovan et al. 2006); the uncertainty of 0.00001 days was negligible for this purpose.

To calculate the relative flux as a function of the projected separation of the planet and the star, we employed the analytic formulas of Mandel \& Agol (2002), using a quadratic limb darkening law,

$$
\frac{I_{\mu}}{I_{1}}=1-u_{1}(1-\mu)-u_{2}(1-\mu)^{2},
$$

where $I$ is the intensity and $\mu$ is the cosine of the angle between the line of sight and the normal to the stellar surface. We chose the values $u_{1}=0.22$ and $u_{2}=0.32$, based on the tabulated values of Claret (2004) and the estimates by Sozzetti et al. (2007) of the stellar effective temperature, surface gravity, and metallicity. We accounted for the color-dependent residual air mass effects with a parameter $k$ specific to each transit, such that the observed flux is equal to the intrinsic (zero air mass) flux times $\exp (-k z)$, where $z$ is the air mass. The best fitting values of $k$ were $0.0021,0.0086$, and -0.0005 , for September 11, September 16, and November 2, respectively. We also fitted for the out-of-transit flux $f_{\text {oot }}$.

The light curves cannot be used to determine both the stellar mass and radius; there is a fitting degeneracy $R_{\star} \propto M_{\star}^{1 / 3}$. Our usual approach is to assume a value for $M_{\star}$ (based on external analyses of the stellar spectrum) and then determine $R_{\star}$ by fitting the light curves. This case was slightly different because we worked in conjunction with Sozzetti et al. (2007), who sought to improve the estimates of the stellar parameters based on the results of the light-curve fit. We worked iteratively, as described below in more detail; for our final analysis, we fixed $M_{\star}=0.98 M_{\odot}$.

Our fitting statistic was

$$
\chi^{2}=\sum_{j=1}^{N_{f}}\left[\frac{f_{j}(\mathrm{obs})-f_{j}(\mathrm{calc})}{\sigma_{j}}\right]^{2},
$$

where $f_{j}(\mathrm{obs})$ is the flux observed at time $j, \sigma_{j}$ controls the relative weights of the data points, and $f_{j}(\mathrm{calc})$ is the calculated value. It is important for $\sigma_{j}$ to include measurement errors and also any unmodeled systematic effects, and in particular to account for time-correlated noise, which effectively reduces the number of independent measurements. Our approach was as follows. First, we rescaled the instrumental uncertainties such that $\chi^{2} / N_{\text {dof }}=1$ for the best fitting model. Table 1 lists the resulting uncertainties. Second, we followed the procedure of Gillon et al. (2006) to decompose the observed noise into "white noise" (that which averages down as $1 / \sqrt{N}$, where $N$ is the number of data points) and "red noise" (that which does not average down over some specified time interval). Specifically, we calculated the standard deviation of the residuals $(\sigma)$ and the standard deviation of the time-averaged residuals $\left(\sigma_{N}\right)$. The averaging time was $1 \mathrm{hr}$ (a timescale comparable to the transit event), corresponding to a number $N$ of data points that depended on the cadence of observations. Then we solved for the white noise $\sigma_{w}$ and red noise $\sigma_{r}$ from the system of equations:

$$
\begin{gathered}
\sigma_{1}^{2}=\sigma_{w}^{2}+\sigma_{r}^{2}, \\
\sigma_{N}^{2}=\frac{\sigma_{w}^{2}}{N}+\sigma_{r}^{2} .
\end{gathered}
$$

Finally, to account approximately for the effective reduction in the number of independent data points, we rescaled the $\sigma_{j}$ in equation (3) by the factor $\sigma_{r} /\left(\sigma_{w} / \sqrt{N}\right)$. In this case, the September 11 and November 2 transits did not show evidence for red noise according to this criterion, but for the September 16 transit the red-noise rescaling factor was 1.14 . For that transit, we find $\sigma_{r}=$ 0.00016 and $\sigma_{w}=0.0014$. To be conservative, we applied this same factor 1.14 to the data from all three transits.

In short, there were 12 model parameters: $\left\{R_{\star}, R_{p}, i\right\}$ as well as $\left\{T_{c}, k, f_{\text {oot }}\right\}$ for each of three transits. We determined the a posteriori probability distributions for these parameters using the same Markov chain Monte Carlo algorithm described in previous TLC papers. We took the median value of each probability distribution to be the best estimate of each parameter and the standard deviation to be the $1 \sigma$ uncertainty. In addition to this statistical error, for the special cases of $R_{\star}$ and $R_{p}$ there is an additional error arising from the uncertainty in $M_{\star}$, which we add to the statistical error in quadrature.

Our choice of $M_{\star}$ merits further discussion, since it is based on a novel iterative procedure conducted in tandem with Sozzetti et al. (2007). The underlying idea is that when fitting a light curve, the results for $R_{\star}$ and $R_{p}$ depend on the choice of $M_{\star}$, while the result for $R_{\star} / a$ is independent of $M_{\star}$, because both $R_{\star}$ and $a$ vary as $M_{\star}^{1 / 3}$ for a fixed value of the orbital period. (There is, however, a minor dependence of $R_{\star} / a$ on the choice of limb darkening function, which is in turn informed by the estimates of the stellar parameters.) Meanwhile, as Sozzetti et al. (2007) have shown, $R_{\star} / a$ is useful for estimating $M_{\star}$, since it can be directly related to the stellar mean density through Kepler's third law (see also Seager \& Mallén-Ornelas 2003):

$$
\frac{a}{R_{\star}}=\left(\frac{G P^{2}}{4 \pi^{2}}\right)^{1 / 3}\left(\frac{M_{\star}+M_{p}}{R_{\star}^{3}}\right)^{1 / 3} .
$$

This makes $R_{\star} / a$ a useful proxy for $\log g$ for the purpose of comparing the observed stellar properties with theoretical isochrones. The advantage of $R_{\star} / a$ is that in typical cases it is more precisely determined than the spectroscopic value of $\log g$.

We iterated as follows: First, we fitted the light curves using the choices $M_{\star}=1.08 M_{\odot}, u_{1}=0.18$, and $u_{2}=0.34$, based on the previous estimates of the relevant stellar parameters by O'Donovan et al. (2006). Next we passed our results for $R_{\star} / a$ to Sozzetti et al. (2007), who used it to refine the estimate of $M_{\star}$. (We refer the reader to Sozzetti et al. [2007] for details on how this refinement was achieved.) In return, Sozzetti et al. (2007) provided us with a new estimate of $M_{\star}$, along with a new estimate of the stellar surface gravity (which affects the choice of limb darkening law). We refitted the light curves using the updated values of the stellar mass and the slightly adjusted limbdarkening law. Then we passed our new result for $R_{\star} / a$ back to Sozzetti et al. (2007), who used it to refine the estimate of $M_{\star}$ and $\log g$, and so forth. This process converged after a few iterations, leading to the final choices for $M_{\star}, u_{1}$, and $u_{2}$ noted above.

While it is possible for the value of the stellar radius that minimizes $\chi^{2}$ to be inconsistent with the theoretical mass-radius relation, in this case we have effectively required consistency with the theoretical mass-radius relation by iterating with Sozzetti et al. (2007).

\section{RESULTS}

The final results are given in Table 2. In addition to the results for the basic model parameters, we have also included in this table a number of interesting derived quantities, such as $a / R_{\star}$ 
TABLE 2

System Parameters of TrES-2

\begin{tabular}{|c|c|c|}
\hline Parameter & Value & Uncertainty \\
\hline$\left(R_{\star} / R_{\odot}\right)\left(M_{\star} / 0.98 M_{\odot}\right)^{-1 / 3} \ldots$ & 1.003 & 0.017 \\
\hline 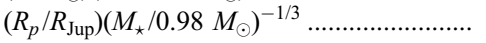 & 1.222 & 0.028 \\
\hline 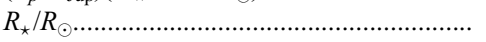 & 1.003 & 0.027 \\
\hline 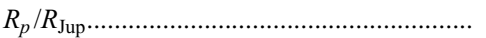 & 1.222 & 0.038 \\
\hline 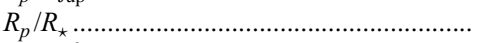 & 0.1253 & 0.0010 \\
\hline$\left(R_{p} / a\right)^{2} \ldots \ldots$ & 0.000270 & 0.000012 \\
\hline$M_{p} / M_{\mathrm{Jup}}{ }^{\mathrm{a}} \cdots$ & 1.198 & 0.053 \\
\hline$T_{\text {eff }}(\mathrm{K})^{\mathrm{a}} \ldots$ & 5850 & 50 \\
\hline$a / R_{\star} \ldots \ldots \ldots \ldots \ldots \ldots$ & 7.63 & 0.12 \\
\hline$i(\operatorname{deg}) \ldots \ldots \ldots \ldots \ldots \ldots$ & 83.57 & 0.14 \\
\hline b.................................. & 0.8540 & 0.0062 \\
\hline 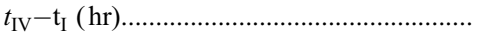 & 1.840 & 0.020 \\
\hline 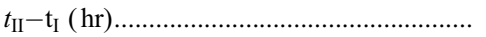 & 0.683 & 0.045 \\
\hline 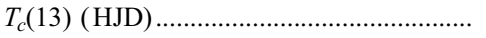 & $2,453,989.75286$ & 0.00029 \\
\hline$T_{c}(15)$ (HJD) & $2,453,994.69393$ & 0.00031 \\
\hline$T_{c}(34)(\mathrm{HJD}) \ldots \ldots \ldots \ldots \ldots$ & $2,454,041.63579$ & 0.00030 \\
\hline
\end{tabular}

NoTES. - The system parameters and their associated uncertainties for TrES-2 are listed; $t_{\mathrm{I}}, t_{\mathrm{II}}$, and $t_{\mathrm{IV}}$, correspond to the times of the first, second, and fourth points when the projected limb of the planet contacts that of the star.

${ }^{a}$ Adopted from Sozzetti et al. (2007).

(which is related to the stellar mean density, as described above) and the calculated durations of the transit and the partial transit phases. The most interesting parameters are the radius of the star, the radius of the planet, the orbital inclination, and the midtransit times, which we discuss in turn.

We find the stellar radius to be $R_{\star}=1.003 \pm 0.027 R_{\odot}$, where the quoted error includes both the statistical error (0.017) and the systematic error due to the uncertainty in the stellar mass (0.021). This estimate agrees with all of the star's observed broadband colors and spectral properties as determined by Sozzetti et al. (2007), as it must, given that our analyses were coupled as described in the previous section.

We find the planetary radius to be $R_{p}=1.222 \pm 0.038 R_{\mathrm{Jup}}$, where (again) the quoted error includes both the statistical error $(0.028)$ and the systematic error due to the uncertainty in the stellar mass (0.026). The difference between our value and the value $R_{p}=1.220_{-0.042}^{+0.045} R_{\text {Jup }}$ presented by Sozzetti et al. (2007) is slight indeed, although our figure has a somewhat smaller error bar. The reason why there is any difference at all is subtle. Sozzetti et al. (2007) determined $R_{p}$ by taking our result for $\left(R_{p} / R_{\star}\right)$ and the associated uncertainty, and multiplying by their estimate for $R_{\star}$ (which in turn was based on matching the observed values of $T_{\text {eff }}, a / R_{\star}$, and metallicity to theoretical isochrones). In contrast, we determined $R_{p}$ and $R_{\star}$ simultaneously by fitting a parameterized model to the light curves, as described above, and then accounting for the uncertainty in the stellar mass. Our analysis takes into account the correlations between all of the parameters, while that of Sozzetti et al. (2007) assumes $\left(R_{p} / R_{\star}\right)$ is independent of $a / R_{\star}$. In this case, our procedure has yielded somewhat more precise results for $R_{p}$ and $R_{\star}$.

For an eclipsing single-lined spectroscopic binary the surface gravity of the secondary $\left(G M_{p} / R_{p}^{2}\right.$ in this case $)$ can be determined nearly independently of any assumptions regarding the properties of the primary (Southworth et al. 2004, 2007; Winn et al. 2007a; Beatty et al. 2007; Sozzetti et al. 2007). This result holds because the fitting degeneracy for the radial-velocity data is $M_{p} \propto M_{\star}^{2 / 3}$ and the fitting degeneracy for the photometric data is $R_{p} \propto M_{\star}^{1 / 3}$, and in the ratio $M_{p} / R_{p}^{2}$ the stellar mass cancels out. There remains only a weak dependency of $R_{p}$ on the choice of

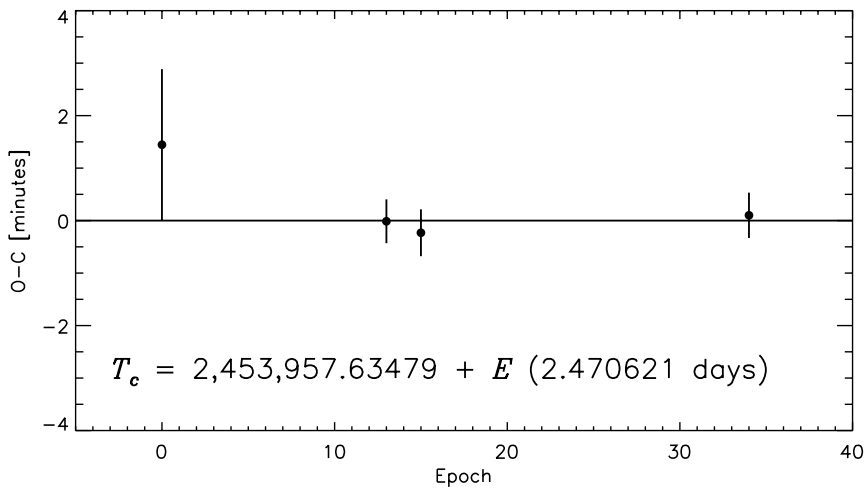

FIG. 2.- Timing residuals (observed - calculated) for four observed transits, according to the ephemeris of eq. (1). The first point corresponds to the $T_{c}$ reported by O'Donovan et al. (2006), and the other three points correspond to the three transits reported in this paper. The points lie on a horizontal line, and therefore the data are consistent with a constant period.

limb-darkening law, which is based on knowledge of the host star. In this case, the result is $G M_{p} / R_{p}^{2}=1976 \pm 91 \mathrm{~cm} \mathrm{~s}^{-2}$, or $\log g_{p}=3.299 \pm 0.020$.

We confirm the finding by O'Donovan et al. (2006) that the transit chord occurs at an unusually large impact parameter, $b \equiv a \cos i / R_{\star}=0.8540 \pm 0.0062$. This is of interest because the error in the impact parameter is much smaller when the impact parameter is high than when the transit is near equatorial (all other things being equal). This facilitates the detection of small changes in the impact parameter due to orbital precession, which can be caused by additional bodies in the system or by the stellar quadrupole field (Miralda-Escudé 2002). A large impact parameter is also advantageous for interpreting the RossiterMcLaughlin effect, as long as an accurate external measurement of the projected rotation speed of the $\operatorname{star}(v \sin i)$ is available (Gaudi \& Winn 2007).

Accurate timing of exoplanetary transits is a promising method for identifying additional planets or moons (see, e.g., Holman \& Murray 2005 and Agol et al. 2005), and in this case transit timing takes on special importance because TrES-2 is in the field of view of the Kepler mission (Borucki et al. 2003; Basri et al. 2005). We have tested whether or not our three measured transit times and the single transit time reported by O'Donovan et al. (2006) are consistent with a uniform period by fitting a linear function of epoch number to the observed times. The residuals to this linear fit are shown in Figure 2 and are consistent with zero within the measurement errors. Thus, there is not yet any indication of timing anomalies. Based on our fit, we have refined the ephemeris. The new value of $T_{c}$ is $2,453,957.63479(38)$ (HJD), and the new value of the orbital period is 2.470621(17) days, where the numbers in parentheses are the $1 \sigma$ uncertainties in the last two digits of each figure.

We conducted two tests to check the robustness of our results. First, we fitted each of the three transits separately and examined the scatter in the results. For each of the parameters $\left\{R_{\star}, R_{p}, i\right\}$, the three different results were all within the $1 \sigma$ uncertainty of the result when fitting all the transits together. Thus, the results of the three transits agree well with one another. Second, we examined the sensitivity of the results to the limbdarkening function, finding also that the results are robust. For example, the effect on $R_{p}$ of allowing the quadratic limb-darkening coefficients to be free parameters (rather than fixing them at the values tabulated by Claret 2004) is an increase of $1.0 \%$. If we use a linear law instead of a quadratic law, $R_{p}$ is increased by $0.6 \%$, and if we use the four-parameter "nonlinear" law of Claret (2004; 
with coefficients fixed at ATLAS-based values), then $R_{p}$ is decreased by $0.5 \%$. None of these changes is very significant compared to the $2.3 \%$ statistical error.

\section{SUMMARY}

Through observations and analysis of three transits, we have improved on the estimates of the orbital and physical parameters of TrES-2. Our results are consistent with the estimates of the stellar and planetary radii by O'Donovan et al. (2006) but have smaller uncertainties. We also show that the available transit times are consistent with a uniform period. In our analysis of the photometry we have made use of an improved estimate of the stellar mass from Sozzetti et al. (2007). This estimate was obtained by iteratively combining values of $a / R_{\star}$ determined from the light curves with values of effective temperature and metallicity determined from stellar spectra. This novel technique can be applied to all transiting systems for which high-quality stellar spectra and high-precision light curves are available. Our obser- vations and analysis help lay the ground work for interpreting the $\sim 600$ transits of TrES-2 that will be observed by Kepler.

We thank E. Falco for accommodating our observing schedule changes. KeplerCam was developed with partial support from the Kepler mission under NASA Cooperative Agreement NCC21390 (PI: D. Latham), and the KeplerCam observations described in this paper were partly supported by grants from the Kepler mission to SAO and PSI. N. M. J. H. acknowledges support for this work NASA Origins grant NG06GH69G. Work by F. T. O'D. and D. C. was supported by NASA under grant NNG05GJ29G, issued through the Origins of Solar Systems Program. A. S. gratefully acknowledges the Kepler mission for partial support under NASA Cooperative Agreement NCC 2-1390. G. T. acknowledges partial support for this work from NASA Origins grant NNG04LG89G.
Agol, E., Steffen, J., Sari, R., \& Clarkson, W. 2005, MNRAS, 359, 567

Basri, G., Borucki, W. J., \& Koch, D. 2005, NewA Rev., 49, 478

Beatty, T. G., et al. 2007, ApJ, 663, 573

Borucki, W. J., et al. 2003, in ASP Conf. Ser. 294, Scientific Frontiers in Research on Extrasolar Planets, ed. D. Deming \& S. Seager (San Francisco: ASP), 427

Burrows, A., Hubeny, I., \& Hubbard, W. B. 2007, ApJ, 661, 502

Charbonneau, D., Brown, T. M., Burrows, A., \& Laughlin, G. 2007, in Protostars and Planets V, ed. B. Reipurth, D. Jewitt, \& K. Keil (Tucson: Univ. Arizona Press), 701

Charbonneau, D., Brown, T. M., Noyes, R. W., \& Gilliland, R. L. 2002, ApJ, 568,377

Charbonneau, D., et al. 2005, ApJ, 626, 523

Claret, A. 2004, A\&A, 428, 1001

Deming, D., Harrington, J., Seager, S., \& Richardson, L. J. 2006, ApJ, 644, 560

Deming, D., Seager, S., Richardson, L. J., \& Harrington, J. 2005, Nature, 434, 740

Dravins, D., Lindegren, L., Mezey, E., \& Young, A. T. 1998, PASP, 110, 610 Gaudi, B. S., \& Winn, J. N. 2007, ApJ, 655, 550

Gillon, M., Pont, F., Moutou, C., Bouchy, F., Courbin, F., Sohy, S., \& Magain, P. 2006, A\&A, 459, 249

Grillmair, C. J., Charbonneau, D., Burrows, A., Armus, L., Stauffer, J., Meadows, V., Van Cleve, J., \& Levine, D. 2007, ApJ, 658, L115

Holman, M. J., \& Murray, N. W. 2005, Science, 307, 1288
REFERENCES

Holman, M. J., et al. 2006, ApJ, 652, 1715 2007, ApJ, 655, 1103

Mandel, K., \& Agol, E. 2002, ApJ, 580, L171

Miralda-Escudé, J. 2002, ApJ, 564, 1019

O’Donovan, F. T., et al. 2006, ApJ, 651, L61

Queloz, D., Eggenberger, A., Mayor, M., Perrier, C., Beuzit, J. L., Naef, D., Sivan, J. P., \& Udry, S. 2000, A\&A, 359, L13

Richardson, L. J., Deming, D., Horning, K., Seager, S., \& Harrington, J. 2007 Nature, 445, 892

Seager, S., \& Mallén-Ornelas, G. 2003, ApJ, 585, 1038

Southworth, J., Wheatley, P., \& Sams, G. 2007, MNRAS, in press (astro-ph/ 0704.1570)

Southworth, J., Zucker, S., Maxted, P. F. L., \& Smalley, B. 2004, MNRAS, 355,986

Sozzetti, A., Torres, G., Charbonneau, D., Latham, D. W., Holman, M. J., Winn, J. N., Laird, J. B., \& O’Donovan, F. T. 2007, ApJ, in press

Vidal-Madjar, A., Lecavelier des Etangs, A., Désert, J.-M., Ballester, G. E., Ferlet, R., Hébrard, G., \& Mayor, M. 2003, Nature, 422, 143

Winn, J. N., Holman, M. J., \& Fuentes, C. I. 2007a, AJ, 133, 11

Winn, J. N., Holman, M. J., \& Roussanova, A. 2007b, ApJ, 657, 1098

Winn, J. N., et al. 2005, ApJ, 631, 1215

2006, ApJ, 653, L69

$2007 \mathrm{c}, \mathrm{AJ}, 133,1828$

Young, A. T. 1967, AJ, 72, 747 